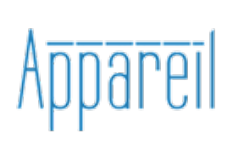

Appareil

$22 \mid 2020$

Une philosophie de l'appareil

\title{
La notion d'appareil chez Jean-Louis Déotte et le dispositif foucaldien
}

\section{Denis Skopin}

\section{(2) OpenEdition}

1 Journals

\section{Édition électronique}

URL : http://journals.openedition.org/appareil/3508

DOI : 10.4000/appareil.3508

ISSN : 2101-0714

Éditeur

MSH Paris Nord

Référence électronique

Denis Skopin, «La notion d'appareil chez Jean-Louis Déotte et le dispositif foucaldien », Appareil [En ligne], 22 | 2020, mis en ligne le 21 décembre 2020, consulté le 26 mars 2021. URL : http:// journals.openedition.org/appareil/3508; DOI : https://doi.org/10.4000/appareil.3508

Ce document a été généré automatiquement le 26 mars 2021.

\section{(c) (i) (3)}

Appareil est mis à disposition selon les termes de la Licence Creative Commons Attribution - Pas d'Utilisation Commerciale - Pas de Modification 4.0 International. 


\title{
La notion d'appareil chez Jean-Louis Déotte et le dispositif foucaldien
}

\author{
Denis Skopin
}

1 J'appartiens à l'école de pensée de Jean-Louis Déotte. Appartenir à une école ne signifie pas marcher sur les chemins déjà explorés, ce n'est pas répéter ce qui est déjà dit ; contrairement à ce qu'affirment ceux qui n'en savent rien, ce n'est pas mettre sa médiocrité à l'abri des dangers que prépare la recherche indépendante (la médiocrité a bien d'autres abris, surtout lorsqu'il s'agit des universités et des universitaires). L'appartenance à une école ouvre des horizons et des pistes à explorer, permet d'aller directement au cœur d'une problématique. Le rapport de maître à disciple n'est pas seulement un rapport de pouvoir, il renferme toujours une donation, un geste de générosité : vous pouvez prendre les choses que vous voulez et les utiliser comme si elles étaient les vôtres.

La philosophie de Jean-Louis Déotte était une philosophie généreuse, qui donnait beaucoup plus qu'elle ne vous "prenait»: loin d'être une théorie rigide voulant soumettre toutes les autres conceptions du monde à la sienne, elle offrait de la place à de nouvelles idées et personnes. Jean-Louis Déotte n'a jamais gardé jalousement ses concepts, mais a été au contraire très attentif et tolérant aux usages que ses disciples, moi compris, faisaient d'eux. Je ne l'ai jamais vu se mettre en position de gardien de ses concepts, ne l'ai jamais entendu parler de leur « distorsion », « usage erroné », etc. Sa théorie des appareils n'était pas une théorie "toute faite», mais un vaste projet de recherche qui accueillait de nouveaux venus; c'était un point de rencontre pour une équipe internationale et interdisciplinaire de chercheurs qui mettaient la technique au cœur de leur questionnement.

D'ailleurs, l'esprit d'ouverture n'était pas la seule chose qui attirait les jeunes chercheurs dans son équipe. Le projet de Déotte, qui consistait à démontrer le profond conditionnement de notre mode d'être et de notre pensée par la technique, était en avance sur la plupart des recherches esthétiques de l'époque. Il s'agissait d'une démarche profondément contemporaine, qui rompait avec l'idée fétichiste et 
préindustrielle de l'art - une démarche encore plus actuelle aujourd'hui qu'il y a vingttrois ans, au moment de la publication de L'Homme de verre ${ }^{1}$.

Bien sûr, s'y ajoutait la fascination exercée par les textes du directeur de l'équipe. Loin d'être limitée à la connaissance des "sources philosophiques", son immense culture couvrait les vastes champs de l'histoire de l'art européen, la littérature, le cinéma, l'histoire des sciences, etc. Sa façon d'aborder les choses - en jetant des ponts entre des domaines qui semblaient fort éloignés - était toujours très élaborée.

5 La notion qui servait à Déotte pour établir les correspondances évoquées, qui lui permettait de découvrir un ordre caché derrière l'apparente hétérogénéité des phénomènes culturels, c'était la notion d'appareil. Bien sûr, le concept d'appareil n'était pas l'unique concept déottien et il fonctionnait en liaison avec quelques autres, comme l'onto-théo-cosmétique, l'immersion ou l'innervation. Pourtant, cette notion restait centrale pour lui et il ne cessait d'y réfléchir dans des contextes différents.

Notre objectif ici sera de confronter l'idée d'appareil, comme elle est développée dans l'œuvre de Déotte, à l'idée de dispositif. Nous nous proposons d'explorer les différences et les proximités des deux concepts en prenant pour exemple le fonctionnement de l'appareil photographique.

6 Bien que les réflexions qui vont suivre soient marquées d'un certain excès terminologique, il ne s'agit pas de substituer à la pensée quelque alchimie linguistique, comme une simple délimitation des notions et la réglementation de leur usage. On sait que la question de la terminologie est loin d'être sans importance pour la philosophie. Les concepts véhiculent la pensée philosophique et sa portée se définit par leur force. Notre objectif ici est donc de penser l'appareil photo et la photographie autrement qu'avec la notion de dispositif.

La notion d'appareil chez Déotte, tout comme celle de dispositif chez Michel Foucault ou de Gestell chez Martin Heidegger, n'est pas univoque. À l'instar du Gestell heideggérien, elle se trouve au centre d'une constellation de concepts: apparaître, appareiller, parer, etc.

7 Il faut dire que la construction de la notion d'appareil est fait par Déotte sous l'influence de sa lecture de Foucault, comme résultat d'une certaine insatisfaction face à la notion de dispositif. Concernant la philosophie du musée que Déotte développait à ce moment-là, et plus largement l'esthétique ${ }^{2}$, l'efficacité de la notion de dispositif se montrait limitée. Celle-ci ne pouvait pas rendre compte de la puissance de l'apparition et de la création : Déotte avoua dans son dernier livre qu'après avoir essayé d'appliquer cette notion dans le domaine de l'esthétique, il ne tarda pas à s'en séparer et à la désigner comme "insuffisante à capter la puissance inaugurale de l'institution muséale $^{3}$ ».

8 Aussi, la notion d'appareil doit-elle beaucoup à la reconsidération critique par Déotte du concept de «surface d'inscription » du maître de Déotte, Jean-François Lyotard. Les appareils paradigmatiques sont le musée, mais aussi la perspective dont les analyses occupent une place importante dans Discours, figure ${ }^{4}$. La notion de parergon chez Derrida a elle aussi joué un certain rôle dans l'élaboration du concept : tout comme le parergon derridien ${ }^{5}$, l'appareil est la condition de ce qui apparaît, ce qui vient à l'être.

Qu'est-ce qu'un appareil ? L'appareil est à comprendre comme un dispositif technique complexe dont les effets s'étendent à plus d'une sphère et se font sentir autant dans le domaine de la technique que dans ceux de l'art, de la science et de la politique. Selon 
Jean-Louis Déotte, « les savoirs et les arts sont toujours appareillés selon des dispositifs techniques époquaux. Au cœur du principe de l'appareil, il y a la fonction de "rendre pareil", d"“apparier" : de comparer ce qui jusqu'alors était hétérogène ${ }^{6}$ ».

Il est important de dire aussi que, un peu à l'instar de l'idée hégélienne, qui est un principe absolu qui se réalise dans une chose, «l'appareil » chez Déotte est un principe abstrait qui se réalise dans un objet technique qui enregistre des traces (ainsi, l'appareil perspectif se "matérialise» en objets techniques concrets comme la boîte perspectiviste de Leon Battista Alberti et le portillon d'Albrecht Dürer). C'est en ce sens que Déotte parle d'un "dispositif d'appareil " ", mais les appareils innervent aussi nos sens, si bien que leur statut peut être dit « mental ».

11 L'originalité de la notion d'appareil est mise en évidence lorsqu'on la confronte à quelques notions voisines qui ont en commun de renvoyer à l'idée d'un conditionnement de l'être humain par la technique. À ce titre, on peut mentionner non seulement la notion de dispositif, dont il sera question plus loin, mais aussi la notion de prothèse chez Bernard Stiegler et les notions de machine et d'outil telles qu'elles sont présentes chez Karl Marx, Gilles Deleuze et Gilbert Simondon.

Selon Bernard Stiegler, contrairement à la doctrine rousseauiste, l'homme n'apparaît pas « tout fait ", il évolue au fur et à mesure avec et par ses instruments. L'hominisation fut rendue possible par les prothèses qui comblèrent un manque initial propre à l'être humain ${ }^{8}$. Or, selon Déotte, les effets de l'appareil sur l'homme résident dans la sphère symbolique, et non pas anthropologique, et se situent donc à un autre niveau que les prothèses stieglériennes: "ce n'est pas une affaire de vision ou d'amplification prothétique de la vue (lunette astronomique, microscopique, etc.), mais de prévision. Ce n'est pas pensable en termes d'anthropologie, mais d'ontologie, parce qu'avec ce type d'appareillage, on entre dans un monde différent ${ }^{9}$." Le milieu technique des appareils, où nous nous trouvons immergés, innerve nos sens, notre vue et notre ouïe.

13 L'appareil est aussi à distinguer de la "machine ", qui constitue le concept le plus important de la philosophie marxiste de la technique ${ }^{10}$. Inséparable de la fabrique, la machine est un moyen de production et n'a d'autres objectifs que la production des biens. Elle se trouve au cœur de la séparation entre le capitaliste et le prolétaire et pour cette raison est déclarée responsable d'aliénation. L'ouvrier devient son "préposé » et accomplit un travail monotone et mal payé.

14 Ce n'est pas le cas de l'appareil. La rationalité de l'appareil n'est pas économique mais plutôt ludique, puisqu'il doit son apparition au plaisir éprouvé par homoludens. Comme le dit Déotte, « ce n'est pas un hasard si la plupart des appareils inventés au cœur de la modernité relèvent plutôt du ludique, du "jouable" : ce sont des accessoires des plaisirs humains, dont le lieu de naissance n'est ni l'atelier ni le laboratoire, mais la baraque foraine. Les appareils relèvent d'une poétique technique qui est déjà au service d'elle-même, d'un plaisir pris à la fonction que décrit Aristote dans la Poétique ${ }^{11}$. "

Tout comme il ne se réduit ni à la prothèse ni à la machine, l'appareil ne se réduit pas au dispositif tel qu'il est conceptualisé par Foucault. Selon Foucault, le dispositif relie des choses qui appartiennent à des registres différents, « des discours, des institutions, des aménagements architecturaux, des décisions réglementaires, des lois, des mesures administratives, des énoncés scientifiques, des propositions philosophiques, morales, philanthropiques ${ }^{12}$.» Le dispositif n'est pas un objet matériel mais un principe abstrait, 
quoiqu'immanent. La prison, le camp militaire, l'atelier, l'usine, etc., ne sont que des lieux où le dispositif s'exerce.

L'origine de ce terme dans les textes de Foucault n'est pas claire. Selon Giorgio Agamben, Michel Foucault aurait emprunté la notion à son directeur de recherche, Jean Hyppolite. Cependant, la généalogie qu'Agamben en trace et qui fait remonter le dispositif à la notion d'économie et à ses aspects théologiques ${ }^{13}$ paraît assez arbitraire et peut facilement faire place à d'autres.

17 Comme on l'a dit plus haut, le dispositif est un principe immanent. On peut donc avancer l'hypothèse selon laquelle Foucault se sert de ce mot pour parler de ce que les penseurs «structuralistes » de sa génération désignaient comme « structure ». Foucault fait également usage des notions de "schéma ", de «machinerie ", d'« agencement », d'« appareil » ou de «technique » - autant de termes souvent interchangeables dans son discours.

18 Enfin, la notion de dispositif peut apparaître chez Foucault comme traduction et redéfinition du Gestell heideggérien. Ce dernier, comme on le sait bien, est ce qui « met le monde à la disposition », bien qu'il s'agisse d'une façon de penser, d'une optique et non pas d'un ordre. La traduction de Gestell par « dispositif » chez François Fédier va dans le sens de cette hypothèse.

Quelle que soit l'origine de cette notion chez Foucault, il est évident que son usage chez l'auteur de Surveiller et punir est inséparable d'une certaine technophobie, ce qui mène le «foucaldien » Agamben à déclarer son refus d'utiliser des objets techniques comme le téléphone portable.

En s'interrogeant sur les rapports entre le dispositif foucauldien et l'appareil, on aurait tort de dire qu'ils n'ont rien à voir. Au contraire, il serait intéressant et important de se demander comment un objet technique, qui peut s'intégrer au dispositif, peut également être un appareil, c'est-à-dire donner lieu à de nouveaux mondes artistiques, scientifiques, politiques, etc. Pour mener ce genre d'interrogation, le cas de l'appareil photographique paraît particulièrement approprié. Puisqu'il apporte de l'ordre dans la réalité brute, la structure et hiérarchise, l'appareil photographique a quelque chose en commun avec le "dispositif " au sens foucaldien. En même temps, il est producteur d'images et, à ce titre, relève du côté des arts plastiques dont le programme, si l'on en croit Friedrich Schiller ${ }^{14}$, est foncièrement émancipateur.

Les théoriciens qui identifient l'appareil photo à un dispositif foucaldien et dénoncent une complicité cachée entre celui-là et le pouvoir ne manquent pas ${ }^{15}$. John Tagg, qui critique la notion de "médium photographique ", semble aller le plus loin dans cette direction en postulant qu'il n'existe pas de "photographie» comme opération technique. Selon lui, il s'agirait de pratiques hétérogènes de capture d'images qui reçoivent leur signification en fonction du dispositif en place. Le potentiel « documentaire » de la photographie serait le résultat de l'association, de l'articulation, de l'appareil photographique et de l'appareil disciplinaire. La considération de la photographie comme connaissance ou témoignage n'obtient tout son sens et ne peut exister qu'au sein d'un paradigme disciplinaire ${ }^{16}$.

21 Aussi, l'interprétation de la photographie comme dispositif est-elle fréquemment marquée d'une technophobie d'inspiration heideggérienne ou plus souvent marxiste. Des auteurs comme Susan Sontag et Harun Farocki attribuent à la photographie une rationalité capitaliste, même si cette rationalité est fort sublimée et si ses effets se 
localisent dans la sphère symbolique. L'intervention d'une photographie sur un objet, l'acte photographique, se pense comme une appropriation de cet objet et une aliénation de son essence.

Les penseurs qui considèrent que l'appareil photo est "libérateur" sont moins nombreux. Jusqu'à récemment, on ne pouvait citer que peu de noms, dont les plus connus étaient ceux de Vilém Flusser, avec son identification très schillérienne de l'appareil à un jouet, et Walter Benjamin, qui cherchait le potentiel libérateur des nouveaux médias en dépit de son engagement marxiste.

Cette distribution n'est pas un hasard, mais reflète la difficulté tout à fait réelle à penser le caractère émancipateur de la photographie et à en donner des exemples assez convaincants. Sans pouvoir développer ce point d'une façon détaillée, nous allons néanmoins essayer de placer la photographie dans une autre optique, celle de l'appareil déottien.

Comme évoqué précédemment, l'appareil et le dispositif ont quelque chose en commun, à savoir: la capacité à synthétiser l'hétérogène. Ce sont toutefois les différences qui importent et qui font que l'appareil et le dispositif sont irréductibles l'un à l'autre, que chacun d'eux obéit à sa propre logique. Plusieurs traits distinguent l'appareil du dispositif ou, mieux, permettent de voir que la théorie des appareils opère des découpages qui ne coïncident pas avec les découpages foucaldiens. Le concept d'appareil, qui renvoie à l'idée d'un conditionnement technique des différentes sphères, peut fonctionner simultanément dans plusieurs champs -esthétique, politique, juridique, ontologique - en permettant alors de traiter des questions qui n'ont jamais vraiment intéressé Foucault, penseur du pouvoir. Ainsi, chaque nouvel appareil instaure une nouvelle temporalité, en s'interposant entre nous et un temps naturel ; il instaure un nouveau rapport entre le corps et la loi ; d'une façon générale, l'appareil structure le sensible qui nous arrive toujours appareillé. S'il faut formuler la différence la plus générale entre l'appareil et le dispositif, on peut dire que les effets de l'appareil sont plus vastes et se situent à un niveau plus élémentaire que ceux du dispositif tel qu'il est décrit chez Foucault.

25 La théorie des appareils nous permet de penser la photographie autrement que ne le faisaient les foucaldiens et les marxistes, en évitant leurs interprétations étroites, concentrées uniquement sur les processus de contrôle, d'appropriation et d'aliénation. L'usage de l'appareil photo en tant que «dispositif» ne constitue qu'un usage au sein de tout un spectre d'usages possibles auxquels il se prête. Il semble qu'en intégrant la photographie dans un "dispositif ", le pouvoir s'approprie ce qui, à l'origine, ne lui appartient pas. Au lieu d'insister sur la connexion intrinsèque entre l'appareil photo et l'appareil d'État, il serait peut-être plus utile de s'interroger sur la façon dont la photographie a été appropriée par le pouvoir ou bien a pu influencer certaines pratiques du pouvoir, les mettre en question, les déplacer, les réorienter, etc.

Il est significatif que Foucault lui-même, dans ses écrits consacrés à la photographie, soit loin de l'identifier à une technique disciplinaire. Tout au contraire, Foucault se penche sur le potentiel éruptif de la photographie, sur sa capacité à briser les codes rigides de la peinture. Il place la photographie du côté du ludique, en lui attribuant une force disruptive, carnavalesque. Selon lui, au XIx siècle, plus précisément vers les années 1860-1880, la photographie a contribué à la frénésie neuve des images, à leur «migration et leur perversion, leur travestissement, leur différence déguisée ${ }^{17}$ ». 
L'intervention de la photographie dans un monde des images dominé par la peinture a provoqué l'hybridation des genres, des techniques et des supports.

Toutes les études qui dénoncent la photographie comme dispositif ont en commun de se référer aux pratiques du $\mathrm{XIX}^{\mathrm{e}}$ siècle, à la photographie judiciaire, anthropométrique ou médicale. La photographie comme dispositif ne peut effectivement appartenir qu'au paradigme disciplinaire. L'intérêt du pouvoir disciplinaire pour la photographie s'explique par le caractère individualisant de celle-ci. Au même titre que les empreintes digitales, la photographie est ce qui permet d'identifier l'individu, ce qui signifie le séparer et l'isoler de la foule anonyme.

En revanche, il est important de remarquer qu'il existe un processus historique de prise en compte de la photographie par le pouvoir disciplinaire, et plus largement un processus d'ajustement intentionnel de l'appareil au dispositif. Il n'existe aucune parenté initiale et naturelle entre l'appareil photo et le dispositif disciplinaire. Leur association est le résultat d'un effort d'adaptation entrepris par quelqu'un (un officiel, un détenteur du pouvoir). Au cours de ce processus, l'appareil, qui existe déjà comme phénomène culturel, s'intègre dans le dispositif, dans une technologie du pouvoir.

Le point historique où cette adaptation, cette association, a lieu se situe dans les années 1880-1890, l'époque d'Alphonse Bertillon et Jean-Martin Charcot. La photographie anthropométrique et coloniale qui apparaît à la même époque est le résultat du même accaparement de l'objet technique par le dispositif dominant. La photographie judiciaire, anthropométrique et médicale opère la "disciplinarisation ", la codification de la photographie elle-même, en imposant un règlement sur sa prise et sur son fonctionnement (face-profil, succession de cadres, etc.). Au cours de ce processus, quelque chose d'essentiel et propre à la photographie se trouve confisqué. Pour devenir aliénante, la photographie elle-même doit d'abord subir une aliénation, être aliénée de son essence d'appareil. Les rapports initiaux entre le photographié, le photographe et le spectateur, à savoir une certaine réciprocité, mutualité et confiance sont confisqués par les rapports de pouvoir.

Néanmoins, la reproductibilité de la photographie se marie difficilement avec la discipline et le contrôle. Cette reproductibilité "contagieuse " va à l'encontre du contrôle en traversant les frontières et transgressant les interdits. C'est la raison pour laquelle les images photographiques elles-mêmes doivent passer par la censure politique et subir toutes sortes de manipulations.

L'ajustement de la photographie aux objectifs disciplinaires nécessite aussi et surtout un travail bureaucratique de documentation qui rend possible le fonctionnement des photos au sein d'une archive policière ${ }^{18}$. La photographie n'est «judiciaire» que lorsqu'elle fait partie d'un dispositif, sans quoi elle redevient une image tout court. Ce n'est pas un hasard si ceux qui essayaient d'utiliser la photographie pour les objectifs judiciaires finissaient par comprendre son inefficacité dans ce domaine. L'entreprise de Bertillon, à savoir d'atteindre un contrôle total en ajustant l'appareil photographique au pouvoir disciplinaire, fut vouée à l'échec exactement à cause de cette inefficacité, de l'utilité limitée de la photographie pour les objectifs de la reconnaissance ${ }^{19}$. Tout porte à croire que la «violence " de la photographie n'est pas une violence propre à l'appareil, mais une violence "extérieure" propre à un dispositif qui s'accapare l'appareil photo et le met à son service pour un certain temps. Comme le rappelle Foucault, le dispositif peut mobiliser des pratiques et des institutions qui à l'origine n'ont rien à voir avec lui. Étant un produit historique, le couplage appareil/dispositif 
n'est pas définitif, ni irréversible. Il existe toujours un point historique où le pouvoir se "désintéresse » d'un appareil et à partir duquel ils se séparent. Ainsi, au xx siècle, la place de la photographie comme instrument de contrôle devient marginale. La fonction de surveillance est transférée à la vidéo, un appareil techniquement plus avancé qui assure un enregistrement continu sans que le sujet surveillé s'en aperçoive.

Si l'exercice de la discipline est inséparable de la visibilité des individus sur lesquels il s'applique et repose sur cette visibilitée ${ }^{20}$, le biopouvoir, qui est toujours massifiant et vise des groupes, doit entretenir avec la visibilité un autre genre de rapports. «L'abandon» de la photographie par le pouvoir (qui d'ailleurs n'est jamais complet) est-il lié à l'émergence du biopouvoir et d'un nouveau paradigme politico-esthétique, celui de la disparition en masse ${ }^{21}$ ? Dans le paradigme disparitionniste, la visibilité de celui qui est voué à disparaître, les traces laissées par la victime, fût-ce les restes de son corps ou les photographies de cette victime, est un fait fâcheux du point de vue du pouvoir. Les lieux de la disparition en masse doivent être autant de trous noirs géographiques, de zones sans noms, de même que le crime politique qui y a eu lieu doit rester secret, inaccessible aux futurs juges et historiens.

Dans ces circonstances, la photographie commence à jouer un autre rôle, en retournant en quelque sorte à ses rapports initiaux avec son référent, c'est-à-dire à sa valeur d'appareil. La prise d'une photo constitue nécessairement une forme d'inclusion, une forme de reconnaissance de ce ou celui qu'on photographie. La reconnaissance du tort subi par les vaincus de l'histoire ne va jamais sans cette inclusion, sans cette visibilité. L'appareil photographique a rendu possible l'inscription de ce qui restait invisible, en redéfinissant le partage du sensible qui existait à cette époque-là. Contrairement à ce qu'affirme Jacques Rancière, le rôle de l'appareil photographique dans l'établissement du « régime esthétique » de l'art était plus considérable que celui de la littérature. C'est la photographie qui a permis aux petites gens et aux petits sujets littéraires, tous les deux " insignifiants ", d'accéder à la visibilité en les rendant dignes de représentation et d'apparition dans l'espace politique. Ce n'est point un hasard si, dans beaucoup de cas, ceux qui subissent un tort préfèrent être photographiés, préférant la photo et un certain « contrôle » qu'elle assume à l'absence totale d'images.

Est-ce pourtant vraiment un contrôle ? Il serait plus correct de dire que le rapport dont il s'agit est légal ou quasiment légal : quelqu'un - qui passe à la photo volontairement, sans y être contraint - confie son corps au photographe qui à son tour le transmet au spectateur. Dans le cas où le photographié subit un tort, il peut faire du photographe un commis de ses souffrances, en le chargeant de la mission de les porter à la connaissance de la partie tierce. De ce point de vue, chaque photographie est un legs, alors que le photographe est un intermédiaire entre le référent et le spectateur, qui, lui, serait un « fidéicommissaire ».

Si l'appareil photo peut être mobilisé pour exercer une violence - laquelle, il faut le rappeler, reste toujours symbolique - cette violence n'est point une violence propre à l'appareil photo en tant que tel. Ce constat vaut également pour les photos des souffrances d'autrui. Si ces photos peuvent être dues aux pulsions sadiques de leur auteur, le sadisme ne constitue pas le propre de l'acte photographique. La prise d'une photo peut avoir beaucoup d'autres motifs, dont le premier et le plus important est l'empathie du photographe pour celui qui souffre. S'il n'intervient pas directement pour aider la victime, le photographe peut néanmoins participer à l'événement «à sa façon », en se portant témoin volontaire de ce qui se passe. 

de violence veulent se mettre en position de témoin. En outre, il existe toujours une distance qui sépare le photographe de l'événement, une certaine passivité du photographe qui n'intervient pas pour protéger celui qui souffre, de façon qu'on aurait tort de le nommer un allié de la victime. Le photographe est séparé aussi bien de la victime que de l'auteur de la violence par une sorte de mur invisible qui empêche la " communication ». Le photographe peut toutefois essayer de s'identifier à la victime, essayer de se placer, fût-ce théoriquement, esthétiquement, en position de la victime. Tout comme le témoin, le photographe peut vouloir parler pour les morts, au nom des morts. Il est capable de prendre la parole là où les vaincus de l'histoire, qui sont morts, ne peuvent pas articuler le tort qu'ils ont subi. Le photographe qui se porte témoin assume une dette envers celui dont il a photographié la mort. Le sentiment de culpabilité qu'il éprouve est très proche de celui du témoin hanté par les spectres des morts, qui ne cesse de déclarer sa dette envers les morts, et toujours commence sa narration par énumérer ceux pour qui il parle. Le souci du témoin qui est un intermédiaire entre les morts et les vivants consiste à jeter un pont entre les uns et les autres, à trouver un langage qui rend possible la médiation entre ces deux communautés. Il va de soi que le témoignage photographique n'est pas équivalent au témoignage oral, mais il doit obéir au même impératif éthique et faire face au même paradoxe épistémologique ${ }^{22}$.

37 En guise de conclusion, on peut ajouter un autre argument en faveur de l'impossibilité de réduire la photographie au dispositif. La théorie des appareils essaie de soustraire la technique à une finalité étroitement politique et économique. À ce point, le projet de Déotte recoupe le projet de Simondon, qui tâche de penser les objets techniques à partir d'eux-mêmes. Pour ce dernier, il s'agit avant tout de cesser de projeter sur les objets techniques la logique qui leur est extérieure, en premier lieu la logique économique. En dernière instance, la rationalité économique contredit la rationalité technique et leurs alliances se trouvent instables. Cette contradiction devient de plus en plus évidente à mesure que la perfection technique de l'objet augmente. Un objet technique avancé n'obéit plus uniquement à la loi du profit. Il constitue un objet ouvert à une "marge d'indétermination" (Simondon) considérable qui ne permet pas de l'assigner à un usage déterminé23.

Dirigée contre la soumission des objets techniques à la logique économique, la critique simondonienne peut être mobilisée contre l'extension sur la technique de la rationalité du pouvoir. On aurait tort de classer l'appareil photo dans la même catégorie d'objets que la guillotine (Phéline), l'arme et la machine (Sontag), la prison, la caserne, etc., et plus largement de le considérer dans la même optique. Tout comme elle n'est pas économique, la logique de l'objet technique n'est pas disciplinaire ou biopolitique, même s'il peut être intégré dans le dispositif. L'évolution technique constitue un processus indépendant et la technicité peut aussi bien entrer en contradiction avec les exigences du pouvoir, s'agit-il du pouvoir souverain, disciplinaire ou du biopouvoir. L'invention technique et la succession des paradigmes techniques ne sont pas liés au changement des paradigmes du pouvoir ${ }^{24}$.

Il est évident que les réflexions ci-dessus n'explorent qu'une piste parmi beaucoup d'autres que nous ouvrent les recherches de Jean-Louis Déotte. Le travail philosophique à faire peut s'inscrire en continuation de la réflexion de Déotte, mais aussi porter sur la place qu'occupe son projet au sein de la philosophie contemporaine. Ainsi, l'analyse 
que nous avons esquissée dans cet article (Déotte-Foucault) peut être complétée par l'analyse comparative de la pensée de Déotte avec la philosophie de Simondon et Heidegger, dont les projets ont également consisté à essayer de combler l'abîme entre la pensée technique et les sciences humaines. Tout en rejoignant ce programme, JeanLouis Déotte pense le rapport entre la technique et les arts autrement que ne le font Simondon et Heidegger, en proposant une toute autre herméneutique historique de ce rapport, qui ne se réduit ni à une histoire de l'oubli de la technique par la culture occidentale, ni à l'idée de sa domination à travers les époques. Ce qui ouvre le thème d'un autre article, d'une autre étude.

\section{BIBLIOGRAPHIE}

Agamben Giorgio, Qu'est-ce qu'un dispositif ?, trad. de l'italien M. Rueff, Paris, Payot \& Rivages, coll. « Rivages poche. Petite bibliothèque », 2007.

Anstett Élisabeth et Dreyfus Jean-Marc (dir.), Cadavres impensables, cadavres impensés. Approches méthodologiques du traitement des corps dans les violences de masse et les génocides, Paris, Pétra, coll. «Les cadavres dans les violences de masse et les génocides », 2012.

Brossat Alain et Déotte Jean-Louis (dir.), L'époque de la disparition. Politique et esthétique, Paris, L'Harmattan, coll. « Esthétiques », 2000.

Deleuze Gilles et Guattari Félix, Capitalisme et schizophrénie, t. 1 L'Anti-๕dipe, Paris, Éditions de Minuit, coll. « Critique », 1972.

Déotte Jean-Louis, L'Époque de l'appareil perspectif. Brunelleschi, Machiavel, Descartes, Paris, L’Harmattan, coll. « Esthétiques », 2001.

Déotte Jean-Louis, L'Époque des appareils, Paris, Lignes, 2004.

Déotte Jean-Louis, L'Homme de verre. Esthétiques benjaminiennes. Paris, L'Harmattan, coll. « Esthétiques », 1998.

Déotte Jean-Louis, Le Musée, l'origine de l'esthétique, Paris, L'Harmattan, coll. « La philosophie en commun », 1993.

Déotte Jean-Louis, Le Passage du musée, Paris, L’Harmattan, coll. « Esthétiques », 2017.

Déotte Jean-Louis, Qu'est-ce qu'un appareil ? Benjamin, Lyotard, Rancière, Paris, L'Harmattan, coll. « Esthétiques », 2007.

Derrida Jacques, La Vérité en peinture, Paris, Flammarion, coll. « Champs », 1978.

Didi-Huberman Georges, Invention de l'hystérie. Charcot et l'iconographie photographique de la Salpêtrière, Paris, Macula, coll. « Macula scènes », 1982.

Foucault Michel, Dits et écrits, 1954-1988, t. II 1976-1988, éd. établie sous la dir. de Daniel Defert et François Ewald, Paris, Gallimard, coll. « Bibliothèque des sciences humaines », 1994.

Foucault Michel, Dits et écrits, 1954-1988, t. III 1976-1979, éd. établie sous la dir. de Daniel Defert et François Ewald, Paris, Gallimard, coll. « Bibliothèque des sciences humaines », 1994. 
Foucault Michel, Surveiller et punir. Naissance de la prison, Paris, Gallimard, 1993.

Lyotard Jean-François, Discours, figure, Paris, Klincksieck, coll. « Esthétique », 1971.

Marx Karl, Le Capital. Critique de l'économie politique, trad. de l'allemand J. Roy, Paris, PUF, coll. « Quadrige », 1993.

Phéline Christian, L'Image accusatrice, Marmande, Association de critique contemporaine en photographie, coll. « Les Cahiers de la Photographie », 1985.

Razac Olivier, Histoire politique du barbelé, nouv. éd. revue et augmentée, Paris, Flammarion, coll. « Champs. Essais », 2009.

Schiller Friedrich, Lettres sur l'éducation esthétique de l'homme [1795], trad. de l'allemand R. Leroux, Paris, Aubier, coll. « Domaine allemand bilingue », 1992.

Simondon Gilbert, Du mode d'existence des objets techniques, Paris, Aubier, coll. « R.E.S. L'Invention philosophique », 1989.

Stiegler Bernard, La Technique et le temps, t. 1 La Faute d'Épiméthée, Paris, Galilée, 1994.

Tagg John, The Disciplinary Frame: Photographic Truths and the Capture of Meaning, Minneapolis, University of Minnesota Press, 2009.

\section{NOTES}

1. Jean-Louis Déotte, L'Homme de verre. Esthétiques benjaminiennes, Paris, L'Harmattan, coll. « Esthétiques », 1998.

2. Jean-Louis Déotte, Le Musée, l'origine de l'esthétique, Paris, L'Harmattan, coll. « La philosophie en commun », 1993.

3. Jean-Louis Déotte, Le Passage du musée, Paris, L'Harmattan, coll. « Esthétiques », 2017, p. 14.

4. Jean-François Lyotard, Discours, figure, Paris, Klincksieck, coll. « Esthétique », 1971.

5. Jacques Derrida, La Vérité en peinture, Paris, Flammarion, coll. « Champs », 1978.

6. Jean-Louis Déotte, Qu'est-ce qu'un appareil? Benjamin, Lyotard, Rancière, Paris, L'Harmattan, coll. « Esthétiques », 2007, p. 11.

7. «Précisons qu'un tel appareil n'est pas réductible à un dispositif, même s'il n'y a pas d'appareil sans dispositif technique, c'est-à-dire sans une certaine configuration concrète, extérieure et intérieure, close, finie, mettant en œuvre, enregistrant, des traces, au sens le plus général, et un support » (Jean-Louis Déotte, Ibidem, p. 51).

8. Bernard Stiegler, La Technique et le temps, t. 1 La Faute d'Épiméthée, Paris, Galilée, 1994.

9. Jean-Louis Déotte, L'Époque de l'appareil perspectif. Brunelleschi, Machiavel, Descartes, Paris, L'Harmattan, coll. « Esthétiques », 2001, p. 24.

10. Karl Marx, Le Capital. Critique de l'économie politique, Paris, PUF, coll. « Quadrige », 1993, livre premier, chapitre 13 «Machinerie et la grande industrie». Voir aussi Gilles Deleuze et Félix Guattari, Capitalisme et schizophrénie, t. 1 L'Anti-đEdipe, Paris, Éditions de Minuit, coll. « Critique », 1972.

11. Jean-Louis Déotte, L'Époque des appareils, Paris, Lignes, 2004, p. 179.

12. Michel Foucault, Dits et écrits, 1954-1988, t. III 1976-1979, éd. établie sous la dir. de Daniel Defert et François Ewald, Paris, Gallimard, coll. «Bibliothèque des sciences humaines », 1994, p. 299.

13. Giorgio Agamben, Qu'est-ce qu'un dispositif?, trad. de l'italien M. Rueff, Paris, Payot \& Rivages, coll. « Rivages poche. Petite bibliothèque », 2007.

14. Friedrich Schiller, Lettres sur l'éducation esthétique de l'homme [1795], trad. de l'allemand R. Leroux, Paris, Aubier, coll. « Domaine allemand bilingue », 1992. 
15. On peut citer ici : John Tagg, The Disciplinary Frame: Photographic Truths and the Capture of Meaning, Minneapolis, University of Minnesota Press, 2009 ; Christian Phéline, L'Image accusatrice, Brax, Association de critique contemporaine en photographie, coll. «Les Cahiers de la Photographie », 1985 ; Georges Didi-Huberman, Invention de l'hystérie. Charcot et l'iconographie photographique de la Salpêtrière, Paris, Macula, coll. « Macula scènes », 1982.

16. John Tagg, op. cit., p. xxvii.

17. Michel Foucault, Dits et écrits, 1954-1988, t. II 1976-1988, éd. établie sous la dir. de Daniel Defert et François Ewald, Paris, Gallimard, coll. «Bibliothèque des sciences humaines », 1994, p. 707.

18. Il en va de même pour l'appareil perspectif qui, trois siècles après son invention, se trouve approprié par le pouvoir disciplinaire qui l'utilise pour distribuer les corps dans l'espace. Selon Déotte, « le panoptique n'est rien d'autre qu'un appareil de perspective ajusté à la discipline des corps ». Jean-Louis Déotte, L'Époque des appareils, op. cit., p. 182.

19. Voir Christian Phéline, L'image accusatrice, op. cit.

20. Michel Foucault, Surveiller et punir. Naissance de la prison, Paris, Gallimard, 1993, p. 173.

21. Alain Brossat et Jean-Louis Déotte (dir.), L'époque de la disparition. Politique et esthétique, Paris, L'Harmattan, coll. « Esthétiques », 2000, p. 13-28.

22. Le rôle du photographe est à rapprocher avec celui du commis et du témoin, mais aussi de l'historien et du conservateur du musée. Comme l'indique Jean-Louis Déotte, la photographie a quelque chose à voir avec le musée qui « sauve » le passé. Par une photo, dans une photo, le passé reste dans le présent. La temporalité qu'instaure la photo n'est pas linéaire, à la différence de la temporalité de l'appareil perspectif.

23. Gilbert Simondon, $\mathrm{Du}$ mode d'existence des objets techniques, Paris, Aubier, coll. «R.E.S. L'Invention philosophique ", 1989.

24. On peut rappeler que les «objets» qui ont fait le plus de morts au $x^{e}$ siècle sont extrêmement simples du point de vue technique et ne doivent rien à l'esprit de l'invention, à la technè (sur les «techniques » de la mise à mort au xx siècle, voir : Cadavres impensables, cadavres impensés. Approches méthodologiques du traitement des corps dans les violences de masse et les génocides, Élisabeth Anstett et Jean-Marc Dreyfus (dir.), Paris, Pétra, coll. « Les cadavres dans les violences de masse et les génocides", 2012.) De la même façon, «l'invention» du fermier américain Joseph Glidden a consisté à utiliser deux fils de fer au lieu d'un, ce qui a permis au barbelé de se tenir (Olivier Razac, Histoire politique du barbelé, Paris, Flammarion, 2009). C'est l'extrême simplicité de ces «techniques » qui a permis de les appliquer à l'échelle industrielle et a assuré leur triste efficacité politique.

\section{RÉSUMÉS}

Cet article aborde les différences et les proximités de deux concepts, ceux de l'appareil chez JeanLouis Déotte et du dispositif chez Foucault.

La notion déottienne d'appareil s'inscrit dans une série de concepts philosophiques (Gestell, machine, prothèse, outil...) qui servent à décrire les différents aspects de la technique moderne et plus largement à penser la place occupée par les objets techniques dans nos sociétés. Bien qu'utilisée dans un autre contexte, la notion de dispositif chez Foucault a elle aussi de fortes connotations techniques, évoquant les aspects techniques du pouvoir d'un côté et décrivant la technique comme intrinsèquement liée au phénomène du pouvoir de l'autre côté. En prenant 
pour exemple l'appareil photographique, nous essayons de montrer que la notion d'appareil donne la possibilité d'établir une autre hiérarchie de la technique et du pouvoir. À la difference du dispositif, le concept d'appareil permet non seulement de penser la rationalité technique comme irréductible à celle du pouvoir, mais aussi d'accentuer la primauté du conditionnement technique par rapport à tous les autres, y compris le conditionnement par le pouvoir. En outre, la notion d'appareil peut servir de base pour une nouvelle herméneutique historique de la technique, qui diffère des modèles qu'on trouve chez Heidegger et Simondon.

\section{INDEX}

Mots-clés : technique, appareil, dispositif, Gestell, prothèse, machine, pouvoir, discipline, biopouvoir

\section{AUTEURS}

\section{DENIS SKOPIN}

Maître de conférences, Université de Saint-Pétersbourg, Faculté d'arts libéraux et sciences ; denis.skopin@mail.ru 